\title{
Activity and enrichment use in disabled Asiatic black bears (Ursus thibetanus) rescued from bile farms
}

\author{
JA Dallaire*t, N Field ${ }^{\ddagger}$ and GJ Mason ${ }^{\dagger}$
}

\author{
† Department of Animal and Poultry Science, University of Guelph, 50 Stone Road East, Building \#70, Guelph, Ontario, Canada NIG 2WI \\ * Animals Asia Foundation, China Bear Rescue Centre, Longqiao, Xindu District, Chengdu, Sichuan, China 610505 \\ * Contact for correspondence and requests for reprints j.ahloy.dallaire@gmail.com
}

\begin{abstract}
Physical disability has the potential to impede the use of environmental enrichments in rehabilitation programmes. We therefore compared the behaviour of 63 disabled and non-disabled socially housed adult Asiatic black bears rescued from bile farms for 103 observation hours. Amputees were less active than non-amputees, spent less time standing, travelled less between different areas of their outdoor enclosure, and showed less frequent stereotypic behaviour. Blind bears also showed low levels of activity and stereotypic behaviour. Blind bears and male amputees spent less time than non-disabled bears eating food dispersed throughout the enclosure as a foraging enrichment. It is unclear whether their infrequent eating is due to impaired foraging, or to lower energy demands arising from lower activity levels. Blind bears tended to manipulate feeders and other enrichment objects less than sighted bears. Disabled bears did not show any signs of impaired social interactions, and were not competitively displaced from resources by other bears more often than non-disabled bears. Thus, disabled bears rescued from bile farms show deficits in overall activity, with amputees also travelling less around their enclosures and blind bears potentially compromised in some forms of enrichment use. However, it is apparent that they adapt well to the presence of social companions. Several disabled bears also showed a degree of novel behaviour, compensating seemingly for disabilities, suggesting possible avenues for enrichments targeted specifically at these bears. The data also suggest specific hypotheses to test in longitudinal studies of rehabilitation.
\end{abstract}

Keywords: animal welfare, Asiatic black bear, blindness, environmental enrichment, limb amputation, physical disability

\section{Introduction}

Over 10,000 captive Asiatic black bears (Ursus thibetanus) are farmed for bile, used in traditional Chinese medicine, in China, Vietnam, and Korea. They typically experience solitary confinement, extreme physical restriction, and malnutrition in addition to bile extraction, every one to three days, through a catheter or fistula (reviewed by Li 2004; Loeffler et al 2007, 2009). These animals' poor welfare is manifest in physical health problems (eg chronic infection) and behavioural changes (eg excessive fear of keepers, abnormal repetitive behaviours, such as self-sucking or head rolling). Bears born in captivity (circa $80 \%$ of rescued bears) are usually also maternally deprived, being weaned at age three months, rather than the 1.5 years common in the wild (Loeffler et al 2007). The Animals Asia Foundation (AAF) is rescuing bears from closed bile farms and taking them to sanctuaries in Vietnam's Tam Dao National Park and in Sichuan Province, China. There, efforts are made to rehabilitate them progressively, eventually culminating in bears being group-housed in large, environmentally enriched enclosures.
While most bears show remarkable recovery, others make slower progress and exhibit behaviour indicative of anxiety or fear, as well as stereotypic behaviour. One possible reason for poor recovery could be little use of the provided environmental enrichments. Environmental enrichment, widely used by animal keepers to improve welfare (eg in zoos), can be defined as the identification and provision of "environmental stimuli necessary for optimal psychological and physiological wellbeing" (Swaisgood \& Shepherdson 2005; see Young 2003, for examples of treatments considered to be enriching). In various species, enrichments have been shown, for example, to decrease anxiety and stress-related corticosterone reactivity (eg Benaroya-Milshtein et al 2004) and to reduce time spent performing stereotypic behaviour (reviewed in Shyne 2006; Swaisgood \& Shepherdson 2006). However, individuals clearly differ in the degree to which they benefit, and stereotypic behaviour reduction can be correlated to strength of motivation to use enrichments (Tilly et al 2010). In the case of the Asiatic black bears at Animals Asia's sanctuaries, an extensive environmental enrichment and management programme is in place, designed to meet 


\section{Figure I}

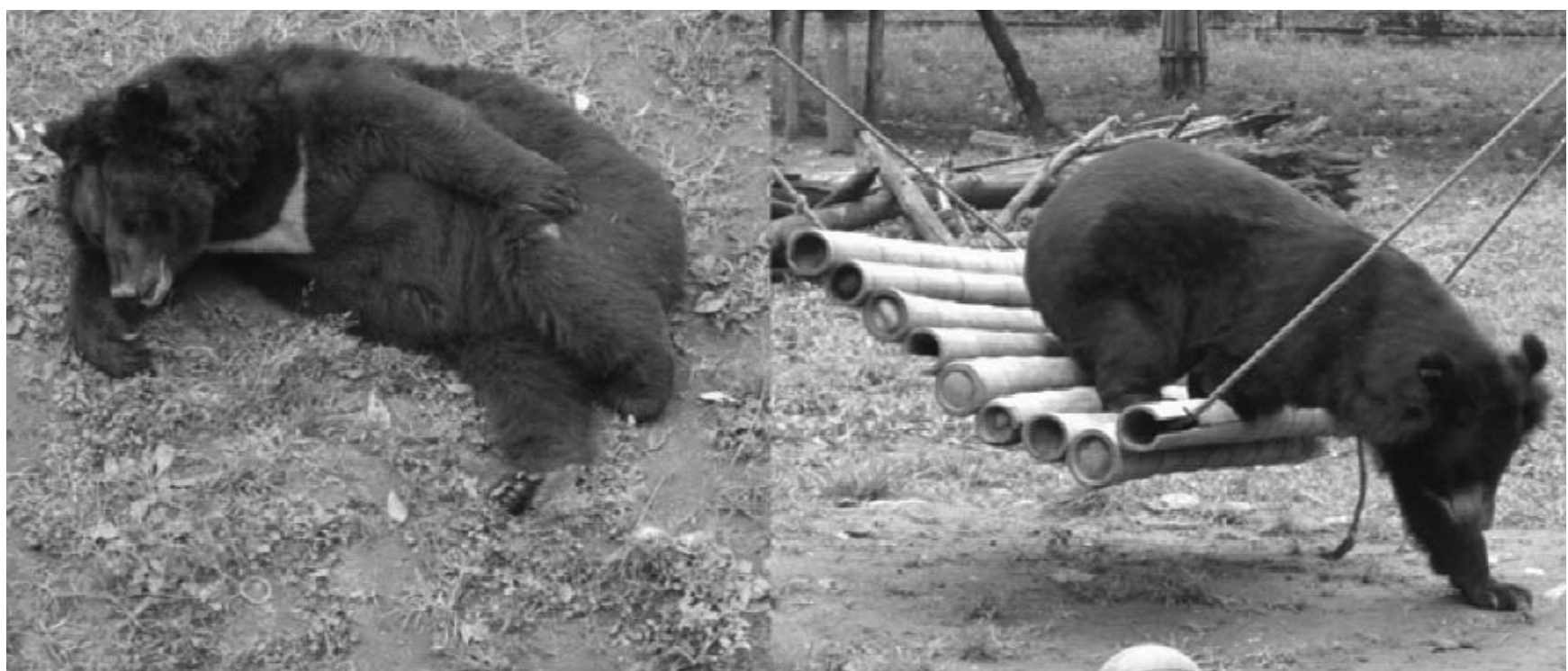

The bear on the left has intact limbs. The bear on the right has had his right forelimb amputated below the elbow.

the complex behavioural and veterinary needs of the bears. They are provided opportunities for social interaction, object manipulation, sensory stimulation, foraging, swimming, and climbing (see Materials and methods), in addition to positive reinforcement from keepers, used for husbandry and preventative medicine purposes.

One group potentially at risk for limited enrichment use, and thence for poor recovery, is physically disabled bears, of which there are a sizable number at Animals Asia's sanctuaries. Nearly a quarter of the sanctuary bears $(23.8 \%)$ have some form of eye disease, and $4.4 \%$ are blind. Ten per cent are missing limbs or paws (Figure 1), mostly as a result of snare injuries (and, in a few anecdotal cases, limbs were amputated from farm bears to make bear paw soup). Many of these amputees may suffer from chronic pain. In one study, $92.5 \%$ of human victims of traumatic, non-clinical amputation developed traumatic neuromas and reported 'stump pain', even several years after the event (Lacoux et al 2002).

Evidence from humans, primates, captive bears, and companion animals suggests that blindness and limb amputation can create impediments to locomotion, foraging, and social interaction. Experimentally blinded infant rhesus macaques (Macaca mulatta) moved about their environments less quickly than did sighted infants, and unlike their sighted counterparts did not approach a feeder provided by the researchers without maternal assistance (Berkson 1973). Similarly, three blind European brown bears (Ursus arctos) in a zoo spent much of their time sleeping and showed limited exploration, to the point that they failed to discover the entrance to a separate part of their enclosure (Koene 1998). Contact with electric fences always caused them to retreat to familiar areas. Human leg amputees (even with prostheses) typically walked more slowly and in a more energetically costly manner than non-amputees (Fisher \& Gullickson 1978), while wild chimpanzees (Pan troglodytes) with severe snare injuries were less proficient at processing food items than were non-amputees (Stokes \& Byrne 2001). In terms of social interactions, young blind children engage in less complex play and in less social interaction than their sighted peers (Troster \& Brambring 1994). Blind infant rhesus macaques similarly initiated fewer play bouts than did sighted infants (Berkson 1973). Blind individuals may have a reduced ability to perceive or produce normal social signals: blind infant rhesus macaques produced some apparently inadvertent, but threatening social signals, sometimes leading to aggression by the receiver (Berkson 1973), and blind infant crab-eating macaques (Macaca fascicularis) less frequently showed facial expressions during social interactions than did sighted infants (Berkson 1977). An infant Japanese macaque (Macaca fuscata) with congenitally missing hands and malformed feet was found in proximity to other infants or juveniles less often than were non-disabled infants, and played with them less often, seemingly because his limited mobility prevented him from following them (Nakamichi et al 1983). Finally, clinical reports of geriatric companion animals further suggest that both blindness and pain may lead to increased aggression (Landsberg \& Araujo 2005).

The above studies also show, however, that animals may adapt to their disabilities by adjusting behaviours or developing entirely novel ones. Thus, wild chimpanzees whose limbs were only moderately injured by snares showed no deficits in food processing because they prioritised suitable techniques (eg using the injured limb for passive object 
support, stripping leaves with lips rather than fingers) (Stokes \& Byrne 2001). Blind rhesus macaques navigated around their environment and located food in part by feeling the ground with their hands (Berkson 1973). The Japanese monkey with missing hands eventually learned to walk bipedally (Nakamichi et al 1983).

We studied a group of adult Asiatic black bears being rehabilitated after they were taken from bile farms. We aimed to determine whether limb-amputated and blind bears are impaired in their ability to use environmental enrichments, compared to able-bodied individuals. The goal was to determine whether any special husbandry modifications are needed in order to improve the welfare and rehabilitation prospects of physically disabled bears. We hypothesised that amputees experience increased difficulty, energy requirements, and/or pain associated with locomotion and climbing, as well as decreased ability in physical confrontations. This predicts that they would be less active, spend less time on their feet, show reduced mobility, food acquisition, and enrichment use (particularly of climbing structures), and diminished success in competitive social interactions. Additionally, we hypothesised that blind bears spend increased time in familiar areas and visit other parts of the enclosure less frequently (eg to avoid accidentally touching electric fences: Koene 1998), have difficulty locating and accessing food or enrichment objects, have decreased ability in physical confrontations, and have problems detecting and interpreting certain visual social cues (eg agonistic gapes). We predicted that they would show more circumscribed use of space, reduced food acquisition and enrichment use, diminished success in competitive social interactions, and less affiliative, more agonistic social interactions. In addition, we measured the amount of time these bears spent performing stereotypic behaviour (SB), because the persistence of this behaviour may be taken to indicate that environmental enrichment has been ineffective (Mason et al 2007). We did not make a directional hypothesis concerning stereotypic behaviour, however, because preenrichment levels for these animals are unknown, and because physical disability could conceivably impede SB directly, independently of welfare.

\section{Materials and methods}

\section{Subjects and housing}

Bears brought to the Sichuan Longqiao Black Bear Rescue Centre, near Chengdu, Sichuan, China, are initially quarantined individually in recovery cages with physical and feeding enrichments, while they are nursed back to relative health (including removal of infected gall bladders for nearly all animals) and allowed to put on body mass. They are then moved to larger, enriched concrete dens equipped with sleeping baskets, where they are gradually introduced to conspecifics through the bars of the den doors. Eventually, pairs and then groups that are deemed compatible (ie not dangerously aggressive toward each other) are allowed to interact freely within a shared space. Finally, approximately a year after arrival at the centre, bears are group-housed in large, environmentally enriched enclosures containing, eg scattered food and climbing structures. Bears subsequently receive regular veterinary examinations and their welfare is closely monitored using an integrative veterinary and behavioural management system. These animals are neither bred nor released into the wild: AAF simply aims to let them live out their lives in a captive environment conducive to good welfare. Some bears whose welfare is too poor must be euthanised either on arrival at the sanctuary or because of deteriorating health. These decisions are made by on-site veterinarians in conjunction with behavioural management staff.

We studied bears group-housed in 'bear houses', each of which has six or seven inter-connected indoor dens in addition to a large, semi-naturalistic outdoor enclosure. Dens are concrete rooms measuring approximately $24 \mathrm{~m}^{2}$ each, containing four elevated metal baskets $(1.8 \times 0.95 \mathrm{~m}$; length $\times$ width), in which bears often rest, in addition to a water trough. Enclosures in the houses studied consist of between 2,727 and $3,272 \mathrm{~m}^{2}$ of grassy terrain containing various climbing/shade structures, a swimming pool, and manipulable objects and feeders (Figure 2). Manipulable objects and feeders are rotated on a daily basis, with each item present no more than once a week. Bears spend the night inside the dens and are allowed into the enclosure daily, from approximately 0915 to $1645 \mathrm{~h}$. Dens remain accessible during 'yard hours', except for a brief cleaning period. Before bears are allowed out in the morning, a large amount of seasonally available fruits and vegetables are placed in the enclosure - on top of climbing structures, inside feeders or woodpiles, etc - to encourage foraging, in quantities varying with season. Dry dog food is also scattered extensively on the ground and on raised structures. Certain fruits and vegetables and other special food items (eg dried yak meat, fish paste, herbs, nuts) are rotated in about once a week as part of the enrichment calendar. Bears are fed an additional meal of dog food, fruit, and vegetables upon returning to the dens for the night. A small number of bears are given supplemental food when deemed necessary to maintain a healthy body mass. Pain or mobility medication, delivered in the den inside blended fruitshakes or inside marshmallows, was given at the start and/or end of yard hours to the few specific bears needing it to mitigate the effects of various conditions (eg chronic musculoskeletal, skin, liver, kidney, or heart disease).

From late May to late June, we studied the residents of five houses, containing between 11 and 18 bears each. Ten bears were excluded because they could not be visually identified reliably, leaving 63 subjects (37 females, 26 males). Of these, 19 were amputees and nine were blind, including one blind amputee. Subjects had been at the sanctuary for anywhere between 16 months and 10 years (median $=5$ years), and veterinary staff estimated that most of these bears were young adults or middle-aged, with only a few elderly animals. Because there were very few exceptions that fell outside of these 'young adult' and 'middleaged' categories, we used a median split, statistically treating all animals as belonging to either one or the other of these 


\section{Figure 2}

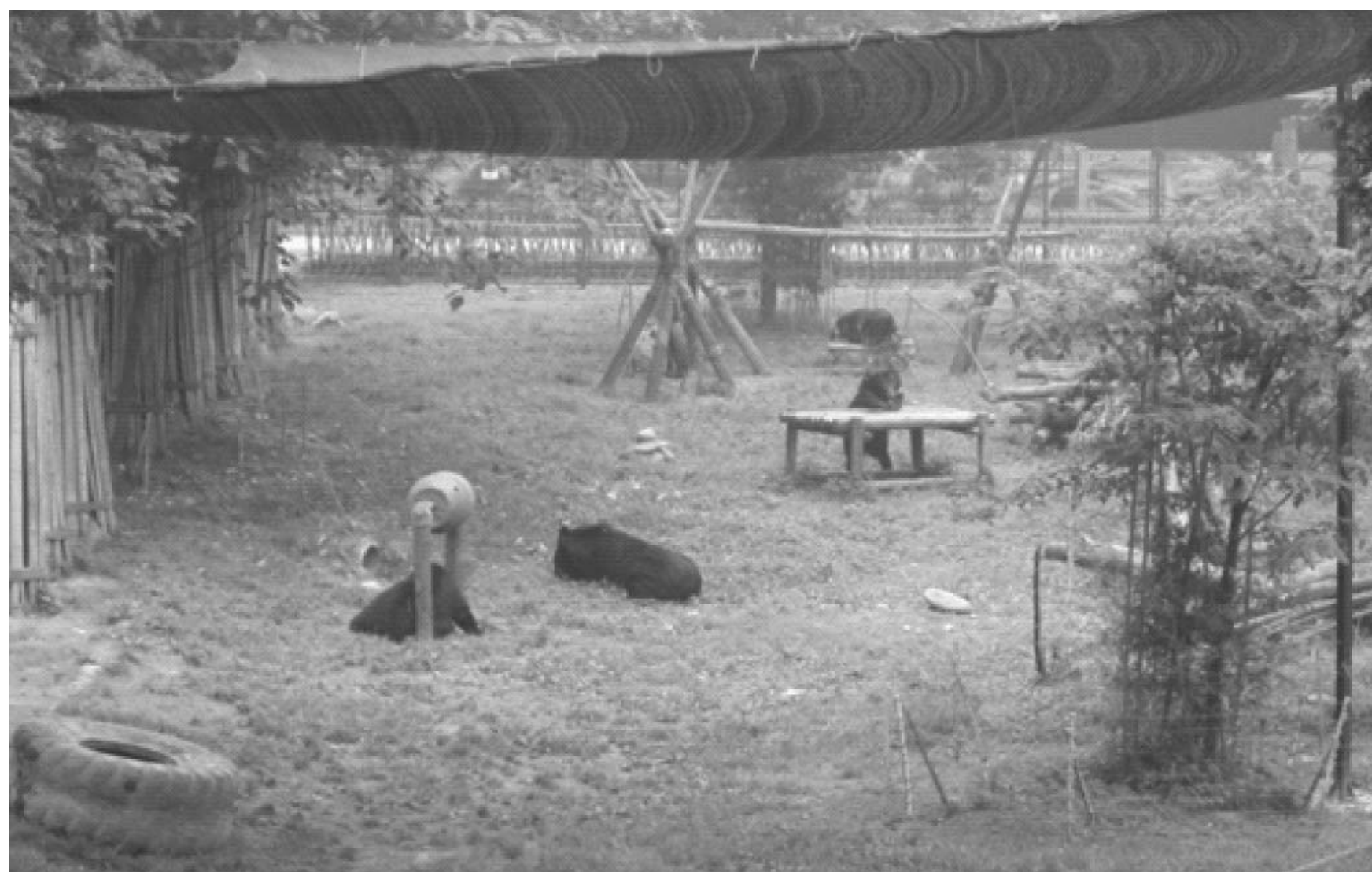

View of one of the bear yards. Environmental enrichments in the picture include a rolling barrel feeder, several different climbing structures, a swing, a large tyre, a digging pit, a log wall with holes for hiding food, and conspecifics.

categories. All males had been castrated upon arrival at the centre. In our sample, disabled bears were no more likely to be taking pain medication than were controls: one of the blind bears $(11 \%)$, three of the amputees $(16 \%)$, and ten of the non-disabled bears $(28 \%)$ received medication daily.

Of the 19 amputees (eleven females, eight males), 12 were missing one forelimb below the elbow (including the blind amputee), two were missing a forepaw, one was missing a forelimb above the elbow, one was missing both a forelimb below the elbow and the other forepaw, two were missing one hind limb below the hip, and one was missing a hind limb below the knee. Of the nine blind bears (four females, five males), one may have had some degree of sight due to a recent operation, while others were obviously completely blind (eg two had no eyes due to prior medical issues).

\section{Behavioural observations}

The observer (JAD) sampled bear behaviour during 'yard hours', when staff are active and the centre is open to visitors, from approximately 1000 to $1200 \mathrm{~h}$ and 1400 to $1645 \mathrm{~h}$. Bears were observed from a rooftop viewing area when they were in the outdoor enclosure and from an area adjacent to their dens when they were inside: both are locations from which bears are accustomed to being observed by staff and visitors. The observer, aided by binoculars, identified bears based on ear tags and physical characteristics. Because locating specific individuals within the enclosure could be very time consuming, the observer used a modified version of scan sampling (Martin \& Bateson 2007) in which he first visually swept the yard from right to left, then the dens, recording each subject's behaviour instantaneously (Table 1), as it was first encountered during a scan. The interval between scans was 15-20 min for houses containing many bears $(16,17$, or 18), and 1015 min for houses with fewer bears (11 and 11). Each house was observed during yard hours for either four or five mornings (24-31 scans) and four or five afternoons (34-49 scans). In addition to the above scan sampling, we also used conspicuous behaviour recording (Martin \& Bateson 2007) of aggressive mounting (outside of play wrestling) and competitive exclusion (see Table 1), recording all observed instances. Finally, because bears performed most of their stereotypic behaviour shortly before the morning and evening feedings (plus outside the closed doors of dens while these were cleaned), we also observed bears inside their dens in the morning ('den hours'), between approximately 0815 and $0915 \mathrm{~h}$, prior to their release into the yard, using five-minute instantaneous scan sampling. The rela- 
Table I Ethogram of relevant behaviours.

\begin{tabular}{|c|c|}
\hline \multirow[t]{3}{*}{ Body position } & On feet: standing, either on all limbs or on hind limbs only, or wrestling \\
\hline & Seated: hindquarters touching the ground or other support (eg climber floor) \\
\hline & Lying: torso touching the ground or other support \\
\hline Climber use & Climbing or otherwise positioned on top of climbing structures, den baskets, posts, woodpiles, etc \\
\hline \multirow[t]{2}{*}{ Activity } & Inactive: seated or lying, either asleep or alert, but doing no more than looking around \\
\hline & Active: standing or seated/lying and engaging in other behaviours (eating, manipulation, etc) \\
\hline \multirow[t]{2}{*}{$\begin{array}{l}\text { Stereotypic } \\
\text { behaviour }\end{array}$} & $\begin{array}{l}\text { Pacing: walking back and forth along the same path three or more times, may include only sideways locomotion } \\
\text { with forelegs while hind legs remain stationary }\end{array}$ \\
\hline & Swaying: swaying/twirling/tossing/jerking head or upper body back and forth $3+$ times, may be combined with pacing \\
\hline Manipulation & $\begin{array}{l}\text { Carrying, biting, pawing, sniffing or otherwise handling objects (eg feeders, toys, branches) with limbs or mouth. } \\
\text { Does not include eating }\end{array}$ \\
\hline Eating & Biting, chewing, swallowing or mouthing a food item (fruit, vegetable, browse, grass or dog food) \\
\hline \multirow[t]{3}{*}{ Social (affiliative) } & Play-wrestling: wrestling in the absence of audible roars or howls. Neither participant attempts to leave the situation immediately \\
\hline & Nuzzling: gentle nosing, sniffing, licking or pawing \\
\hline & Clucking: tongue-clucking vocalisation by subject facing a nearby recipient \\
\hline \multirow[t]{3}{*}{ Social (agonistic) } & Fight: wrestling, swatting, biting, chasing or gaping in the presence of audible roars or howls \\
\hline & $\begin{array}{l}\text { Mounting: one subjects makes ventral-dorsal contact with another, outside the context of play-wrestling. Often } \\
\text { involves biting from top partner and/or howls and escape attempts from the bottom partner }\end{array}$ \\
\hline & $\begin{array}{l}\text { Competitive exclusion: fight (as above) with a clear winner and loser. Loser is the subject which is either aggressively } \\
\text { displaced from a resource it is currently using (eg pool, basket, food), denied access to a resource it approaches, } \\
\text { has an affiliative contact attempt aggressively rebuffed or is otherwise made to flee }\end{array}$ \\
\hline Social (neutral) & Proximity: subjects within circa I $\mathrm{m}$ of each other but not engaging in affiliative or agonistic interactions \\
\hline
\end{tabular}

tively high bear density within the dens, coupled with low lighting and frequent movement by subjects, made it impossible to quickly identify all subjects. The observer therefore identified and recorded only those bears performing SB on a given check. Each house was observed during den hours for between four and eight mornings (46-93 scans). The methods used in this study complied with the requirements of the Canadian Council on Animal Care.

\section{Statistical analyses}

To facilitate the evaluation of mobility and space use, each yard was visually divided into six or nine sectors $(3 \times 2$ or $3 \times 3$ grid, depending on yard size) of relatively equal size, the boundaries of which were identifiable using visual landmarks, and all dens were, together, counted as one additional sector. To evaluate mobility, we counted the number of instances in which a subject was found in different sectors on consecutive scans. We also corrected this measure for overall activity, re-calculating it as a proportion of observations in which bears were not inactive. The extent and evenness of space use was assessed using the Shannon index, typically used to quantify species diversity (Pianka 1966), on the number of times each bear was observed in different sectors. The formula for this index is: $-\Sigma^{s}\left(\mathrm{p}_{\mathrm{i}} \ln \mathrm{p}_{\mathrm{i}}\right)$, where $s$ is the total number of sectors and $\mathrm{p}_{\mathrm{i}}$ is the proportion of scans spent in a given sector. A high Shannon index, in this case, indicates that a bear visits all sectors and spends more or less even amounts of time in each. A low Shannon index indicates that a bear spends the majority of its time in one or just a few sectors, and rarely visits others. Thus, our mobility measure is an index of the amount of time a bear spends travelling within the yard, while our space use measure indicates whether a bear makes frequent use of all areas of the yard, or instead restricts its travel, however frequent, to the same few areas.

Analyses were run in Minitab 15 and used a significance level of $\alpha=0.05$. To test for the effects of each type of disability on behavioural time budgets, we used a General Linear Model (GLM) with Type II (Adjusted) SS, in which 'bear house' was included as a random factor, along with whether a bear had been tapped for bile or not, date of arrival at the sanctuary, whether a bear was taking pain medication or not, body mass, age category, sex, blindness (or lack thereof), limb amputation (or lack thereof), and the interactions of blindness and limb amputation with sex. Where necessary, behavioural frequencies (as proportions of time budgets) were arcsine-square-root transformed to meet assumptions of normality. A few variables (opportunistic counts of social behaviours) did not meet the assumptions required for parametric testing: their distributions included too many zeros, resulting in non-normally distributed GLM residuals. Therefore, we used an alternative statistical procedure in order to test the potential effects of disability on these variables. Each disabled bear was pair-matched to a non-disabled control 
Table 2 Behavioural comparison of amputees and non-amputee controls.

\begin{tabular}{|c|c|c|c|}
\hline & Amputees & Controls & Effect of disability \\
\hline Activity & $41.5-46.2 \%-50.8$ & $52.2-56.4 \%-60.7$ & $F_{1,48}=9.76, P=0.003$ \\
\hline On feet & $25.3-29.3 \%-33.3$ & $34.1-38.5 \%-42.9$ & $F_{1,48}=7.53, P=0.008$ \\
\hline Mobility (number of scan pairs) & $31.4-37.1 \%-42.8$ & $40.7-44.8 \%-48.8$ & $F_{1,48}=5.04, P=0.029$ \\
\hline Mobility (\% of active scan pairs) & $48.8-57.0 \%-65.2$ & $59.4-63.5 \%-67.5$ & $F_{1,48}=2.82, P=0.1$ \\
\hline Space use (Shannon index) & $1.36-1.49-1.62$ & $1.54-1.61-1.68$ & $F_{1,48}=3.37, P=0.072$ \\
\hline \multirow[t]{3}{*}{ Eating } & F: $0.2-8.0 \%-15.9$ & $F: 11.9-14.4 \%-16.8$ & $F_{1,48}=2.94, P=0.093$ \\
\hline & M: $9.4-13.4 \%-17.4$ & M: $10.7-13.8 \%-16.9$ & $\mathrm{~F}: F_{1,25}=0.26, P=0.612$ \\
\hline & & & $M: F_{1,14}=13.34, P=0.003$ \\
\hline Dog food/grass as percentage of diet & $40.9-50.9 \%-60.9$ & $54.4-60.6 \%-66.9$ & $F_{1,48}=3.13, P=0.083$ \\
\hline Manipulation & $2.4-4.1 \%-6.9$ & $4.2-5.1 \%-5.9$ & $F_{1,48}=1.29, P=0.262$ \\
\hline On climber & $23.7-27.8 \%-32.2$ & $18.5-20.9 \%-23.4$ & $F_{1,48}=2.51, P=0.120$ \\
\hline Social contact & $19.3-21.2 \%-23.0$ & $18.8-20.6 \%-22.5$ & $F_{1,48}=0.04, P=0.847$ \\
\hline Affiliative contact & $4.7-6.1 \%-7.6$ & $5.6-7.0 \%-8.5$ & $F_{1,48}=0.60, P=0.443$ \\
\hline Agonistic contact & $1.1-1.6 \%-2.2$ & $1.3-1.7 \%-2.2$ & $F_{1,48}=0.01, P=0.907$ \\
\hline Mounted (bottom partner) & $0-0-1$ & $0-1-3$ & $W_{9}=8, P \leq 0.098$ \\
\hline Competitive exclusion & $0-1-1$ & $0-2-5$ & $W_{12}=12, P \leq 0.233$ \\
\hline Yard hours SB & $1.1-1.6 \%-2.2$ & $1.3-1.7 \%-2.2$ & $F_{1,48}=0.16, P=0.691$ \\
\hline Den hours SB & $2.2-3.3 \%-4.6$ & $8.2-10.2 \%-12.3$ & $F_{1,48}=7.89, P=0.007$ \\
\hline
\end{tabular}

Values are shown as means, surrounded by upper and lower bounds of their $95 \%$ confidence intervals. These are corrected means, derived from the residuals of the GLM model including all parameters except for amputation. For non-parametric tests, values are instead shown as QI - median - Q3. Where analyses were conducted separately for each sex due to a significant interaction between sex and amputation, values are also shown separately. The rightmost column shows the results of statistical tests for a main effect of amputation.

individual of the same sex, within the same enclosure, whenever such a control individual was available (15 amputee/non-amputee matches, seven blind/sighted matches). Disabled bears were matched to controls by three individuals who were not otherwise involved in the project, and who were therefore blind to the behaviour of the subjects. Each did this independently, and majority rule was used where consensus was not reached. Their instructions were primarily to attempt to minimise withinpair differences in body mass, age, and date of arrival at the sanctuary, and secondarily to attempt to match bears based on whether or not they had been previously tapped for bile, and their bile farm of origin. The goal of this procedure was to eliminate the effects of potential confounds, where these could not be controlled for statistically. For the variables tested in this manner, we compared disabled and non-disabled bears using Wilcoxon matched-pairs signed rank tests, which were run by hand, or, when this test was impossible to perform (due to too many tied ranks), we binarised the variables (ie scored them simply as presence or absence of a behaviour) and analysed the potential effects of disability on their prevalence using Fisher's exact tests.

\section{Results}

Amputees were less active and spent less time on their feet than controls during yard time (Table 2, Figure 3). Amputees also showed reduced mobility, but this difference was only a trend if mobility was controlled for activity levels. Amputees also tended to have a lower Shannon index of space use than non-amputees. Thus, amputees were less frequently active and on their feet, moved between different sectors less often overall, and tended to distribute their time between these sectors in a less extensive, less even way. Amputees also tended to spend less time eating than controls, and there was a significant interaction of limb amputation (or lack thereof) with sex $\left(F_{1,48}=5.73\right.$, $P=0.021)$. There was no significant difference among females, but amputee males ate significantly less than control males. We also tested whether amputees ate a higher proportion of easily accessible food (grass and dog food: accessible almost everywhere at ground level) as opposed to foods that seem to be more challenging to obtain (fruit, vegetables, and browse: usually clustered around feeders or climbers, and often requiring manipulation or climbing to access). We found a trend in the direction opposite to that expected: amputee diets tended to consist of less easily 
Corrected means ( \pm SEM), derived from the residuals of the GLM model including all parameters except for amputation and blindness. Both limb-amputated and blind bears were significantly less active than able-bodied controls.

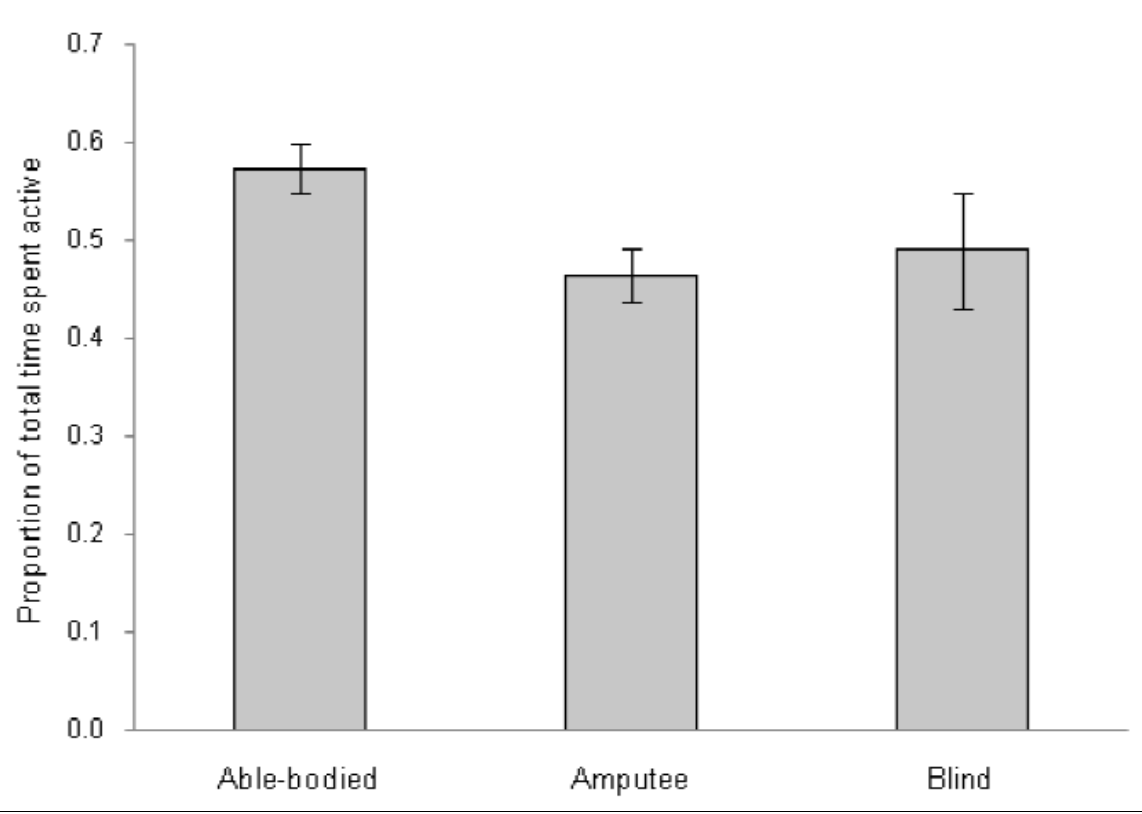

Table 3 Behavioural comparison of blind and sighted controls.

\begin{tabular}{|c|c|c|c|}
\hline & Blind & Controls & Effect of disability \\
\hline Activity & $36.9-46.2 \%-55.5$ & $51.1-54.5 \%-58.0$ & $F_{1,48}=5.47, P=0.024$ \\
\hline On feet & $22.0-32.0 \%-42.0$ & $32.9-36.3 \%-39.8$ & $F_{1,48}=1.4 \mathrm{I}, P=0.240$ \\
\hline Mobility (number of scan pairs) & $27.8-39.5 \%-51.1$ & $39.6-43.0 \%-46.3$ & $F_{1,48}=0.89, P=0.35 \mathrm{I}$ \\
\hline Mobility (percentage of active scan pairs) & $44.2-58.7 \%-73.3$ & $58.4-62.0 \%-65.6$ & $F_{1,48}=0.61, P=0.438$ \\
\hline Space use (Shannon index) & $1.33-1.50-1.67$ & $1.52-1.59-1.65$ & $F_{1,48}=1.49, P=0.227$ \\
\hline Eating & $7.5-9.3 \%-11.4$ & $12.5-13.4 \%-14.3$ & $F_{1,48}=6.10, P=0.017$ \\
\hline Dog food/grass as percentage of diet & $38.4-52.8 \%-67.1$ & $52.8-58.5 \%-64.2$ & $F_{1,48}=0.93, P=0.340$ \\
\hline Manipulation & $2.3-3.4 \%-4.4$ & $4.1-5.0 \%-5.9$ & $F_{1,48}=3.35, P=0.073$ \\
\hline On climber & $18.2-23.0 \%-28.2$ & $20.7-22.9 \%-25.2$ & $F_{1,48}=0.00, P=0.981$ \\
\hline Social contact & $14.8-19.6 \%-24.8$ & $19.6-21.0 \%-22.4$ & $F_{1,48}=0.22, P=0.645$ \\
\hline Affiliative contact & $2.4-5.5 \%-8.6$ & $5.8-7.0 \%-8.2$ & $F_{1,48}=1.34, P=0.252$ \\
\hline Agonistic contact & $0.5-1.5 \%-3.1$ & $1.4-1.7 \%-2.0$ & $F_{1,48}=0.10, P=0.757$ \\
\hline Mounted (bottom partner) & $0-3-6$ & $0-2-4$ & Fisher's exact: $P=1.000$ \\
\hline Competitive exclusion & $1-2-3$ & $0-2-3$ & Fisher's exact: $P=1.000$ \\
\hline Yard hours SB & $0.5-1.2 \%-2.1$ & $1.2-1.6 \%-2.2$ & $F_{1,48}=0.24, P=0.629$ \\
\hline Den hours SB & $0.8-2.4 \%-4.8$ & $7.3-8.9 \%-10.5$ & $F_{1,48}=7.04, P=0.011$ \\
\hline
\end{tabular}

Values are shown as means, surrounded by upper and lower bounds of their $95 \%$ confidence intervals. These are corrected means, derived from the residuals of the GLM model including all parameters except for blindness. For non-parametric tests, values are instead shown as QI - median - Q3. The rightmost column shows the results of statistical tests for a main effect of blindness.

accessible foods. There were no significant differences between amputees and controls in the amount of time spent manipulating feeders or other objects, or in the amount of time spent on top of climbing structures. There was no significant difference in the frequency of social contact in general (affiliative, neutral, and agonistic combined), nor specifically for affiliative or agonistic interaction. Based on opportunistic observations of agonistic interactions, amputees tended to be mounted less often than controls, but there was no difference in the frequency with which they were excluded competitively. Finally, amputees performed only approximately one-third as much stereotypic behaviour as non-amputees during den hours, but there was no significant difference in SB during yard hours. 
Blind bears spent significantly less time active than sighted bears (Table 3, Figure 3). However, unlike for amputees, there was no difference in the amount of time both groups spent on their feet. Neither did blind bears show limited mobility or make significantly less extensive and even use of space than controls. Like amputees, blind bears also spent less time eating than controls, but grass and dog food did not account for a higher proportion of their diet. Blind bears tended to spend less time manipulating objects and feeders, but no less time on top of climbing structures, than sighted bears. There was no significant difference in the frequency of social contact in general, or of any particular type of social interaction, including mountings and competitive exclusion. Blind bears performed less than one-third as much stereotypic behaviour as sighted controls during den hours, but there was no significant difference in SB during yard hours.

\section{Discussion}

In support of our hypotheses concerning amputees, the results suggest that these bears showed a generalised deficit in their levels of activity, including spending less time on their feet and, perhaps partly as a consequence of this, travelling between different areas of the enclosure less frequently. They also showed reduced food acquisition but, in contrast to what we had predicted, did not seem impaired in terms of the use of various enrichments. Neither did they show any evidence of being disadvantaged in social interactions: instead, they actually tended to be aggressively mounted less often than controls. Amputees also tended to use different areas of the enclosure less extensively and evenly than non-amputees, and performed less stereotypic behaviour during den hours, but this was not necessarily to be expected from our hypotheses.

In support of some of our hypotheses concerning blind bears, these animals spent significantly less time eating than sighted bears, and tended to manipulate objects less frequently. Contrary to our expectations, however, they did not show reduced space use or spend less time on top of climbing structures than sighted bears. Neither did they show any significant differences from sighted bears, in terms of the frequency or type of their social interactions. Blind bears showed significantly reduced activity, and low levels of stereotypic behaviour during den hours, which was not necessarily expected. Given that there were only nine blind bears in this study (vs 19 amputees), low power may have prevented us from finding other relationships that would have been significant with a larger sample.

Male amputees and blind bears of both sexes showed both infrequent activity and low amount of time spent eating, compared to controls. We therefore ran an exploratory analysis to test whether activity level and food intake were correlated: across all bears, regardless of disability, time spent eating and time spent active were positively correlated $\left(F_{1,47}=5.11, P=0.028\right)$. Our current results cannot help determine whether disabled bears spent less time eating because their energetic requirements were lower (because of low activity) or due to a reduced ability to obtain food, which might in turn have limited activity. This could be tested by examining whether disabled bears compensate for reduced feeding during yard hours by increasing intake during their second daily meal, delivered inside the dens and not requiring extensive foraging. A small minority of bears (within our sample, two male amputees only) are given extra food individually, during this second meal, in order to counteract loss of body mass. Testing experimentally whether such targeted food supplementation increases activity levels in disabled bears would be another potential way of clarifying the causal relationship between food intake and activity. Given that disabled bears' diets did not include larger proportions of easy-to-access foods, we suspect that disabled bears do not have impaired foraging ability, but rather that low activity precedes and causes low food intake in disabled bears. This proposition now requires further testing.

It is also quite possible that a third factor (eg chronic pain or medical conditions, such as liver cancer) is independently responsible for both inactivity and decreased appetite. There may also have been different causes for low activity in amputees and in blind bears. Amputees spent less time on their feet than controls, but blind bears did not, suggesting that standing and walking may be energetically costly and/or painful or uncomfortable in the former group. While animals in obvious pain are already treated daily, it may be that lower levels of pain or discomfort existing in other individuals have not been detected, and testing whether activity and time spent on feet increase following the start of a course of pain medication could, in the future, help determine whether this is the case.

Why there was a trend toward more restricted use of space in amputees than in non-amputees is unknown. Activity or mobility per se should not be confounds, because the Shannon index is simply sensitive to time spent in different sectors, not to the frequency of movement between sectors. Perhaps extensive and varied use of space is stimulated by feeding motivation, as part of foraging: in this case, low space use could be an artefact of reduced foraging in disabled bears.

Blind bears showed infrequent manipulation of enrichment objects, as expected. Further focal observations should be undertaken to reveal whether this is because these bears approached or encountered these objects less frequently than sighted bears, or because they encountered them often but manipulated them for only short periods of time (eg due to lack of ability or interest). If the former is true, then enrichment use could probably be encouraged in blind bears by facilitating access, but this would be unlikely to work if the latter is true. Amputees, meanwhile, showed the same level of enrichment-object manipulation as non-amputees. One possible reason is that amputees are almost universally wildcaught, as evidenced by their snare wounds, while most of the non-amputees were likely bred on bile farms. Early experience in the wild, eg with foraging and climbing, probably affected enrichment use in these animals. This represents a theoretical confound, although it is somewhat irrelevant from a practical standpoint, given that as far as we know, there are no captive-born amputees at the Animals Asia sanctuary.

Disabled bears unexpectedly performed less stereotypic behaviour than controls, during den hours. This difference 
remains significant even when activity levels are controlled for (using activity levels during yard hours, because activity could not be tracked during den hours: see Materials and methods). If infrequent SB is truly not a consequence of infrequent activity, we do not know how to explain the low levels of SB seen in blind bears. There are some possible explanations for the infrequent SB seen in amputees, however. First, most if not all amputees were likely captured from the wild, and wild-caught animals are likely to have more normally functioning brains that are inherently less prone to repetition, than captive-bred animals (Jones et al 2011). Perseveration, the tendency to inappropriately repeat behaviour, is positively correlated to time spent performing SB in Asiatic black bears (Vickery \& Mason 2003). Second, pacing may have been suppressed in amputees simply because it is painful or excessively energetically costly. While nearly three-quarters of amputees performed more swaying SB (which does not require walking) than pacing SB during morning den observations, this proportion was just over half in non-amputees. The difference, however, was not significant (Fisher's exact test: $P=0.311)$. Whether pacing is suppressed in amputees could be addressed by comparing the effect of pain medication on its frequency in amputees and controls, or by seeing if it is reliably promoted by wet den floors, which may reduce its energetic cost (see below).

The above explanations suggest that interpreting levels of stereotypic behaviour as an indicator of differential welfare in disabled vs non-disabled bears would be somewhat näive. Our observations of stereotypic behaviour also cannot tell us about the degree to which rehabilitation has succeeded, or to which welfare has improved, because we lack data on SB at the start of rehabilitation. A longitudinal study of newly arrived bears is needed to properly track the progression of SB or of other welfare correlates. For the moment, our only grounds for comparison is a study of individually caged, wild-caught Asiatic black bears, housed in a Thai government facility after being confiscated, mostly from the wildlife trade (Vickery 2003). Bears in that study spent, on average, over $40 \%$ of their pre-feeding time performing stereotypic behaviour, compared to slightly over $10 \%$ in our sample. That former bile bears show such low levels of stereotypic behaviour, compared to wild-caught animals which were never farmed, suggests that the rehabilitation process at the Animals Asia sanctuary is, overall, extremely successful.

Aside from the cross-sectional nature of the study, there is another major caveat with regard to these results. They are based exclusively on behavioural observations taken in late spring and early summer, but informal observations by AAF staff suggest that bears at the sanctuary show sizeable seasonal fluctuations in levels of activity, food consumption, and stereotypic behaviour. For example, stereotypic behaviour has been observed to peak in the spring. Seasonal changes in activity, food consumption, and body mass have been observed in this species both in captivity and in the wild, even in the southern parts of its range, where hibernation is uncommon (as it is at the Animals Asia sanctuary) (Reid et al 1991; Hashimoto \& Yasutake 1999; Hwang \&
Garshelis 2007). Further observations are needed to determine whether our behavioural comparison of disabled and able-bodied bears can be generalised across seasons.

Finally, over half (55\%) of the bears at the sanctuary have damaged teeth, usually broken or missing canines, and the question as to whether they are impaired because of this now also requires further study. A pilot project conducted prior to our own research did not find any evidence that canine removal impedes social interactions (H Bacon, AAF's former Veterinary Director, personal communication 2009), but other types of behaviour have not been analysed. Though several of the bears in our sample had damaged teeth, we chose not to control statistically for this variable because the source of the damage cannot be reliably determined on an individual basis. Stereotypic bar-biting is one frequent cause of tooth damage in bile-farmed bears; certain individuals may therefore have damaged teeth because they tend to perform stereotypic behaviour or to be highly active overall. In these cases, tooth damage would be a consequence, rather than a cause, of behavioural characteristics, and controlling statistically for this variable could therefore be inappropriate. Many, but not all, of the bears with damaged teeth have had them removed, which also may affect behaviour and introduce a further complication.

Despite the observed deficits in activity and in some forms of enrichment use, we also observed several behaviours indicative of flexible adaptability in disabled bears. One blind bear, for example, appeared to use tactile feedback to guide her pacing around the circa $1 \mathrm{~m}$ wide concrete strip surrounding the swimming pool: whenever her paw touched the neighbouring grass, she would immediately turn slightly toward the pool before proceeding, thus effectively staying on the concrete strip. It may be that blind bears also used similar cues to avoid electric fences, which were bordered mostly by a similar concrete strip: no bear was seen touching an electric fence. A blind bear was also seen using his paws to probe for footholds while climbing, where other bears would simply have looked for them. Several forelimb amputees moved along the floor of the den, on occasions when water had been spilled on it, by sliding their remaining forepaw along its slippery surface while pushing with their back legs, usually while pacing. Subjectively, this seems much less ungainly than their usual gaits - either hopping along on only one forepaw, or stepping on the tip of their stump as if it were a paw. Some of the most proficient climbers were amputees, who seemed to have very little trouble holding onto structures (or tree trunks) by squeezing them with partially amputated forelimbs, despite the lack of claws. These novel adaptations could potentially be exploited to adjust environmental enrichments to the needs of disabled bears. For example, tactile cues could be placed on the ground between foraging and manipulative enrichments, forming a circuit that could be readily followed by blind animals. Strips of concrete doused with water could similarly facilitate locomotion between enrichments for amputees. 


\section{Animal welfare implications}

Disabled bears showed infrequent activity and stereotypic behaviour, and limited food intake in the outdoor enclosures, as well as reduced use of manipulable enrichments. If the time these animals spend engaging in these behaviours is suboptimal, relative to their motivation to do so, then this may be a cause of poor welfare compared to able-bodied controls. This may be the case if locomotion is too painful and/or costly for limb-amputated bears, if obtaining food is too difficult for either type of disabled bear, or if manipulating feeders or other objects is too difficult or not pleasurable for blind bears. Expanding the use of pain medication and targeted-food supplementation are two potential ways of both testing whether these explanations are valid, and of remedying existing welfare problems. Some individuals showed novel behavioural modifications, which may have compensated for limb amputation and blindness, to some degree, by facilitating behaviours that were otherwise impossible for disabled bears. We recommend that novel enrichments be created to take advantage of flexible adaptations already shown by disabled bears.

\section{Acknowledgements}

We wish to thank the staff at Animals Asia, who were tremendously welcoming and helpful to JAD. We are grateful to Jill Robinson, Angela Leary, and two anonymous reviewers for their helpful comments on the manuscript. Kati Loeffler connected the authors and made this project possible. UFAW, the Ontario Veterinary College Animal Welfare Club, and the Keyes Family Scholarship funded JAD's travel to China.

\section{References}

Benaroya-Milshtein N, Hollander N, Apter A, Kukulansky T, Raz N, Wilf A, Yaniv I and Pick CG 2004 Environmental enrichment in mice decreases anxiety, attenuates stress responses and enhances natural killer cell activity. European Journal of Neuroscience 20: 134I-I347. http://dx.doi.org//0.1 I I //j.|4609568.2004.03587.x

Berkson G 1973 Social responses to abnormal infant monkeys. American Journal of Physical Anthropology 38: 583-586. http://dx.doi.org/I0.1002/ajpa.133038027I

Berkson G 1977 The social ecology of defects in primates. In: Chevalier-Skolnikoff S and Poirier FE (eds) Primate Bio-social Development: Biological, Social, and Ecological Determinants PP 189. 204. Garland Publishing: New York, USA

Fisher SV and Gullickson G 1978 Energy cost of ambulation in health and disability: a literature review. Archives of Physical Medicine and Rehabilitation 59: 124-I33

Hashimoto Y and Yasutake A 1999 Seasonal changes in body weight of female Asiatic black bears under captivity. Mammal Study 24: 1-6. http://dx.doi.org//0.3106/mammalstudy.24.I Hwang MH and Garshelis DL 2007 Activity patterns of Asiatic black bears (Ursus thibetanus) in the Central Mountains of Taiwan. Journal of Zoology 27I: 203-209. http://dx.doi.org/I0.1 I I I/j. I4697998.2006.00203.x

Jones MA, Mason GJ and Pillay N 201 I Correlates of birth origin effects on the development of stereotypic behaviour in striped mice, Rhabdomys. Animal Behaviour 82: I49-159. http://dx.doi.org/10.1016/j.anbehav.2011.04.010

Koene $\mathbf{P} 1998$ Adaptation of blind brown bears to a new environment and its residents: stereotypy and play as welfare indicators. Ursus 10: 579-587
Lacoux PA, Crombie IK and Macrae WA 2002 Pain in traumatic upper limb amputees in Sierra Leone. Pain 99: 309-312 Landsberg G and Araujo JA 2005 Behavior problems in geriatric pets. Veterinary Clinics: Small Animal Practice 35: 675-698

Li PJ 2004 China's bear farming and long-term solutions. Journal of Applied Animal Welfare Science 7: 71-79. http://dx.doi.org/I0.1207/s|5327604jaws070I_5

Loeffler K, Robinson J and Cochrane G 2009 Compromised health and welfare of bears farmed for bile in China. Animal Welfare 18: 225-235

Loeffler K, Robinson J and Cochrane G 2007 Compromised health and welfare of bears in China's bear bile farming industry, with special reference to the free-dripping bile extraction technique. Animals Asia Foundation: Hong Kong, China

Martin P and Bateson P 2007 Measuring Behaviour: An Introductory Guide, Third Edition. Cambridge University Press: Cambridge, UK

Mason G, Clubb R, Latham N and Vickery S 2007 Why and how should we use environmental enrichment to tackle stereotypic behaviour? Applied Animal Behaviour Science 102: 163-188. http://dx.doi.org/ I0.1016/j.applanim.2006.05.041

Nakamichi M, Fujii H and Koyama T 1983 Behavioral development of a malformed infant in a free-ranging group of Japanese monkeys. Primates 24: 52-66. http://dx.doi.org/ 10.1007/BF02381453

Pianka ER 1966 Latitudinal gradients in species diversity: a review of concepts. The American Naturalist 100: 33-46. http://dx.doi.org// 0.1086/282398

Reid D, Jiang M, Teng Q, Qin Z and Hu J 199I Ecology of the asiatic black bear (Ursus thibetanus) in Sichuan, China. Mammalia 55: 22I-238. http://dx.doi.org/10.1515/mamm. 1991.55.2.22।

Shyne A 2006 Meta-analytic review of the effects of enrichment on stereotypic behavior in zoo mammals. Zoo Biology 25: 317-337. http://dx.doi.org//0.1002/zoo.20091

Stokes EJ and Byrne RW 200 I Cognitive capacities for behavioural flexibility in wild chimpanzees (Pan troglodytes): the effect of snare injury on complex manual food processing. Animal Cognition 4: II-28 Swaisgood RR and Shepherdson DJ 2005 Scientific approaches to enrichment and stereotypies in zoo animals: what's been done and where should we go next? Zoo Biology 24: 499-518. http://dx.doi.org// 0.1002/zoo.20066

Swaisgood RR and Shepherdson D 2006 Environmental enrichment as a strategy for mitigating stereotypies in zoo animals: a literature review and meta-analysis. In: Mason $G$ and Rushen J (eds) Stereotypic Animal Behaviour: Fundamentals and Applications to Welfare PP 121-152. CABI: Wallingford, UK. http://dx.doi.org// 0.1079/9780851990040.0256

Tilly SLC, Dallaire J and Mason GJ 2010 Middle-aged mice with enrichment-resistant stereotypic behaviour show reduced motivation for enrichment. Animal Behaviour 80: 363-373. http://dx.doi.org//0.1016/j.anbehav.2010.06.008

Troster $\mathbf{H}$ and Brambring M 1994 The play behavior and play materials of blind and sighted infants and preschoolers. Journal of Visual Impairment and Blindness 88: 42I-432

Vickery SS 2003 Stereotypic Behaviour in Caged Bears: Individual and Husbandry Factors. Wolfson College: Oxford, UK

Vickery SS and Mason GJ 2003 Behavioral persistence in captive bears: implications for reintroduction. Ursus 14: 35-43

Young RJ 2003 Environmental Enrichment for Captive Animals. Universities Federation for Animal Welfare: Wheathampstead, UK. http://dx.doi.org// 0.1002/978047075/046 